the introduction of anti-depressant drugs it still remains the mainstay of the treatment of severe depressive illness, although in recent years it has been used less frequently and with more discrimination.

Over-enthusiasium on the one hand, and suspicion of such a blunderbuss technique on the other have rightly led to a re-evaluation of its place in psychiatry both by the profession and by an increasingly sophisticated public which has become sceptical of medical mystique and inadequately researched empirical treatments.

This collection of papers certainly goes some way towards an objective appraisal of ECT. It offers a wide-ranging review of the historical, clinical, neurophysiological, technical, ethical and research aspects. Contributions have been skilfully edited to produce a readable, stimulating and informative survey of the topic. The unbiased reader (if such a disinterested student exists) will be drawn to conclude that ECT has the qualified approval of the majority of the (admittedly biologically orientated) authors. Coupled with reviews of the all-too-scant literature and reports of original studies it would seem that ECT, properly given, is a safe, effective treatment for attacks of 'endogenous' depression. The less widely accepted role of ECT in the management of schizophrenia is examined and some authors cautiously conclude that the duration of the acute psychosis may be significantly shortened when ECT is used in combination with phenothiazines. Although some patients complain of persistent memory defects (which may not necessarily be due to ECT, but to other co-incidental causes) there is little evidence that average numbers of treatments cause other than a transitory memory disturbance or irreversible brain damage.

Does ECT really work or is it an elaborate placebo? Do differences in technique of administration matter? Is the convulsion the definitive therapeutic ingredient? What effect does it have on brain cells and neuro-transmitters? Is it ever ethically justified to give ECT against a patient's will? Honest attempts are made to begin to answer these questions and areas for further research are opened up.

This book should be required reading for MRC Psych. candidates but, alas, the price (over $6 p$ a page!) may deter other than merit-rewarded consultants, libraries or reviewers from actually owning a copy.

\section{Familial Hyperbilirubinemia}

Edited by L. OkolicsanYI. Proceedings of the Workshop on Familial Disorders of Hepatic Bilirubin Metabolism -Venice, May 1980. Pp. xvii+263, illustrated. John Wiley, Chichester, New York, Brisbane and Toronto, 1980. $£ 15.50$.

The content of this book is the proceedings of a workshop on Familial Disorders of Hepatic Bilirubin Metabolism held in Italy in 1980. The chapters include papers of clinical, biochemical and investigative interest. There is considerable variability in the quality of the individual contributions. If bilirubin is your interest then this book is for you, if not I wouldn't buy it. I do feel however that it should be available in a medical school or hospital library as it contains much useful relevant information on the subject

\section{The Johns Hopkins Atlas of Human Functional Anatomy}

Text edited by G. D. ZuIDEMA; illustrated by L. Schlossberg. 2nd edn, revised and expanded. Pp. 115. Johns Hopkins University Press, Baltimore and London, 1981. $£ 5.95$ (paper), $£ 10.50$ (cloth).

This book provides a thumb-nail sketch of anatomy in the course of 107 pages. Anatomy is correlated with function $\cong$ and thus the section on endocrine glands includes a list of the $C$ hormones produced by the glands. I suspect the chapter on the ear will be found to be rather heavy going. A strong point? of the book is the numerous plates, all in 'glorious techni-O colour'. However, the view of the posterior aspect of the scapula (p. 15) showing the infraspinatus and teres minor muscles is either wrong or shows a very unusual anomaly! $\vec{\nabla}$

The book is useful as an introduction to anatomy for@ medical and dental students and also for nurses, physiotherapists and other paramedical workers.

With a price of less than $£ 6$ for the soft back version, the $\overrightarrow{0}$ book provides surprisingly good value.

\section{Microbial Diseases. Notes, Reports, Summaries, Trends}

Edited by Carl W. May. 1980 edn. Pp. xii +322 , illus 3 . trated. William Kaufmann, Los Altos, California, 1980. $\$ 7.95$ (paperback); $\$ 14.00$ (hard back).

As its title suggests, this work is a compilation of data on communicable diseases which have been drawn largely from. the weekly reports issued over the previous 2 years from the $P$ Centre for Disease Control, Atlanta. The 40 chapters cover the entire spectrum of microbial disease from gonorrhoea? to Rocky Mountain spotted fever, with the inclusion of topics of current interest such as Legionnaires' disease and_ viral haemorrhagic fevers.

The editor's intention is in each case to provide up-to-dateo information on the various diseases both of an epidemiological and more general nature. Most reports are ac- 3 companied by a pertinent editorial comment and a few relevant references. The concept of presenting all the materoal in this form is to be commended; however, the book's imp i t $\overrightarrow{0}$ is somewhat reduced by the incomplete coverage of any ofeco topic. Although published mainly for readers in the United. States, more details on world-wide trends would have helped to put the data in better perspective particularly for workers outside America.

The book should be of interest to both microbiologists and epidemiologists for mostly reference purposes.

\section{Surgical Review 2}

Edited by J. S. P. Lumley and J. L. Craven. Pp. vii +435 , illustrated. Pitman Books, London, 1981. £16.95.

This is the second volume of what promises to be a continuing and highly successful series of interesting surgical essays. $\frac{}{3}$ These are designed, as the editors promise us in their preface, to 'present the personal views of a team of invited experts over a wide range of subjects of surgical interest'.

The 19 chapters can be grouped into specialized topics-i head injuries, lung cancer, the management of unequal legs $\frac{0}{3}$ and coronary arterial surgery; general surgery-piles, varicose veins, testicular and pancreatic tumours, aortic은 aneurysm, gallstones, inflammatory bowel diseases and intestinal stomas-and an interesting group of topics written by non-surgical authorities. These cover interventional angiography (a rapidly advancing and exciting $N$ field), the diabetic patient, radiotherapy of gastrointestinal cancer and the ultrasonic assessment of vascular disease. $N$ There is also a useful contribution on surgical training in the N
Common Market.

The chapters are easy to read, well illustrated, nicely produced and provided with full bibliographies. This isco definitely a book to recommend to every surgeon as $a \frac{\bar{\Phi}}{\Phi}$ useful up-date on current surgical problems. It will be of equal appeal to consultants and to trainees and the editors, we hope, will continue their valuable contribution towards, postgraduate education with future Surgical Reviews. 